\title{
Tanshinone IIA attenuates estradiol-induced polycystic ovarian syndrome in mice by ameliorating FSHR expression in the ovary
}

\author{
JING JIN $^{1^{*}}$, QIAO-YUN HU ${ }^{2 *}$, WEN-WEN XU ${ }^{1}$, WEN-JIA ZHU ${ }^{3}$, BEI LIU ${ }^{1}$, \\ $\mathrm{JING} \mathrm{LIU}^{1}$, WEI WANG ${ }^{1}$ and HUI-FANG ZHOU ${ }^{1}$ \\ ${ }^{1}$ Department of Gynecology, Affiliated Hospital of Nanjing University of Chinese Medicine, Nanjing, Jiangsu 210029; \\ ${ }^{2}$ Department of Pharmacology, School of Basic Medical Science, Nanjing Medical University, Nanjing, Jiangsu 211166; \\ ${ }^{3}$ School of Life Sciences, Nanjing Normal University, Nanjing, Jiangsu 210046, P.R. China
}

Received April 19, 2018; Accepted February 27, 2019

DOI: $10.3892 /$ etm.2019.7352

\begin{abstract}
Tanshinone IIA (TSIIA) is a major component of Salvia miltiorrhiza, a Chinese herb that exhibits a therapeutic effect on polycystic ovary syndrome (PCOS). The present study replicated PCOS via the neonatal treatment of estradiol in mice. Estrous cycles, body and ovarian weight, serum levels of testosterone and estradiol were determined. Histological examination of ovaries was performed. The mRNA and protein levels of aromatase luteinizing hormone receptor and follicle-stimulating hormone (FSHR) in ovaries and granule cells were assayed by reverse transcription-quantitative polymerase chain reaction and western blotting, respectively. TSIIA was revealed to reverse all disorders induced by estradiol treatment, including prolonged estrous cycles, increased body and ovarian weight, increased atretic cyst-like follicles and decreased corpus luteum, large antral follicles and preovulatory follicles. These improvements in PCOS as a result of TSIIA treatment are likely due to the revised testosterone/estradiol balance, as TSIIA reversed the decrease in aromatase mRNA, the enzyme that converts androgen to estrogen. As the expression of aromatase is regulated by the FSH pathway, TSIIA-mediated elevation in FSHR expression may lead to the upregulation of aromatase. Therefore, TSIIA revises the balance of androgen and estrogen by rescuing the reduced expression of FSHR and aromatase, thus attenuating murine PCOS. The current study aimed to further the application of natural drugs in the treatment of PCOS to confront the side effects of hormone drugs and expand the use of TSIIA.
\end{abstract}

Correspondence to: Professor Hui-Fang Zhou, Department of Gynecology, Affiliated Hospital of Nanjing University of Chinese Medicine, 155 Hanzhong Road, Nanjing, Jiangsu 210029, P.R. China E-mail: zhouhuifang2011301@163.com

\section{${ }^{*}$ Contributed equally}

Key words: tanshinone IIA, reproductive disturbances, polycystic ovary syndrome

\section{Introduction}

Polycystic ovarian syndrome (PCOS) is the most common endocrine and metabolic disease in women, occurring in $\sim 15 \%$ at procreation age (1). Its characteristics include hyperandrogenism, ovulation failure and polycystic ovarian morphology (2), combined with multiple clinical symptoms including menoxenia, acne, hirsutism, alopecia, central obesity and infertility (3). PCOS, is a life-long disease, which not only causes short-term problems, including infertility due to anovulation, but also exhibits long-term metabolic issues including obesity, insulin resistance, non-alcoholic fatty liver disease, type 2 diabetes mellitus and cardiac vascular disease (4), as well as hyperinsulinemia and estrogen dependent cancer (5).

Hyperandrogenism, occasionally combined with hypoestrogenism, is a classic diagnostic criterion for PCOS, as excess androgen induces the dysfunction of the hypothalamic-pituitary-ovarianaxis, which is theneurohormonal system that regulates gonadal hormone balance, follicle maturation and ovulation (6). Mainstream therapy including hormonal therapy, antiandrogens and insulin-sensitizing drugs used to reduce androgen levels is usually combined with oral contraceptives (7). Among them, diane-35 (a combination of ethinyl estradiol and cyproterone acetate) is commonly used to treat PCOS (8).

However, diane-35 produces significant hormonal side effects and negatively impacts triglyceride metabolism and pancreatic function (9).

Salvia miltiorrhiza ( $S$. miltiorrhiza), a Chinese herbal medicine, has been used to treat PCOS. Components isolated from $S$. miltiorrhiza, including cryptotanshinone, attenuate PCOS by reversing dexamethasone-induced ovarian insulin resistance in mice (10). However, whether other components of S. miltiorrhiza have the capability to treat PCOS remains unclear. Tanshinone IIA (TSIIA) is another primary component of S. miltiorrhiza (11). Serving as an antioxidant, anti-inflammatory and immunomodulatory element, it is primarily used to treat cardiovascular diseases (12). TSIIA also inhibits oxidative stress injury in neuronal cells to treat Alzheimer's disease (13). The results of the present study confirmed the therapeutic effect of TSIIA on PCOS induced by neonatal 
exposure to estradiol in mice by regulating follicle-stimulating hormone (FSH) receptor (FSHR) expression in the ovary.

\section{Materials and methods}

Animals. Animal care and experiment procedures were performed in accordance with the guidelines of and approved by the Animal Care and Ethical Committee of Affiliated Hospital of Nanjing University of Chinese Medicine (Nanjing, China). A total of 57 female (C57Bl/6J x A/J) F1 (B6A) 5-day-old mice (weight, 4-5 g) purchased from Beijing Vital River Laboratory Animal Technology Co., Ltd. (Beijing, China) were used in the current study. All mice were maintained in standard cages under a $12 \mathrm{~h}$ light/dark cycle at a temperature of $22-24^{\circ} \mathrm{C}$ and a relative humidity of $55-65 \%$, with ad libitum access to food and water.

Experimental design and drug administration. Estradiol (Sigma-Aldrich; Merck KGaA, Darmstadt, Germany) dissolved in dimethyl sulfoxide and diluted with sesame oil (BSZH Co. Ltd., Beijing, China), was subcutaneously injected into 5-day-old female mice (PCOS mice, $n=56$ ) at a dose of $20 \mu \mathrm{g} /$ day for 3 days (14). Vehicle of the same volume was administrated to mice (control group, $\mathrm{n}=28$ ). On the 56th postnatal day, half PCOS mice (estradiol+TSIIA group, $n=28$ ) received TSIIA (Nanjing Spring and Autumn Biological Engineering Co. Ltd, Nanjing, China) for 4 weeks at a dose of $100 \mathrm{mg} / \mathrm{kg} /$ day by gavage. The PCOS mice left (estradiol group, $\mathrm{n}=28$ ) and control mice received the same weight-based volume of vehicle. Estrous cyclicity was determined during the last 18 days prior to sacrifice. Thereafter, mice were weighed (22-25 g) and sacrificed on the 96th postnatal day by decapitation.

Assessment of estrous cycles. Estrous cycles were examined daily at 0800-0900 h. The fluid obtained by vaginal lavage with $0.9 \%$ saline was spotted thinly on a microscope slide. Following air-drying, slides were stained with toluidine blue $(0.1 \%)$ at room temperature for 2 sec. According to the types of vaginal epithelial cells present (leukocyte, nucleated and cornified cells), diestrus, estrus and proestrus were identified using light microscopy at magnification, x100 as previously described (15).

Ovarian histology. Ovaries were excised from mice, fixed in $4 \%$ paraformaldehyde at room temperature for $48 \mathrm{~h}$, dehydrated in ascending grades of ethanol and embedded in paraffin in 5- $\mu \mathrm{m}$ sections. Samples were then deparaffinized, rehydrated and stained with hematoxylin and eosin (H\&E) for 3 and $0.5 \mathrm{~min}$ at room temperature, respectively. Follicles were counted using a conventional light microscope (Olympus DP70; Olympus Corporation, Tokyo, Japan) with x40 objective. The number of follicles (atretic cyst-like, large antral and preovulatory follicles) were counted in every sixth section ( $30 \mu \mathrm{m}$ apart) and multiplied by 6 to provide the total number of follicles in each ovary. Only follicles containing an oocyte with a visible nucleus were counted to avoid double counting. The classification of follicular stages was made following the morphological criteria as described previously $(16,17)$. The number of corpora lutea was scored in a blinded fashion using one section per ovary and one ovary per mouse (18).

Hormonal measurements. Serum was obtained when mice were sacrificed following the determination of estrous cyclicity. Levels of FSH, luteinizing hormone (LH), progesterone (P), estradiol and testosterone (T) were quantified using ELISA kits (cat. nos. E0830Mu, E0441Mu, E0459Mu, E0461Mu and E0458Mu, respectively; Uscn Life Sciences, Inc., Wuhan, China) according to the manufacturer's protocol.

Reverse transcription quantitative polymerase chain reaction $(R T-q P C R)$. Total RNA was extracted from murine ovary tissue using a TRIzol reagent kit (Invitrogen; Thermo Fisher Scientific, Inc., Waltham, MA, USA) according to the manufacturer's protocol. RNA $(1 \mu \mathrm{g})$ was transcribed into cDNA using a PrimeScript ${ }^{\mathrm{TM}}$ RT reagent kit (Takara Biotechnology Co., Ltd., Dalian, China). qPCR reactions were performed using a Light Cycler Fast Start DNA Master SYBR-Green I kit and an ABI Prism 7300 Sequence Detection System (Applied Biosystems; Thermo Fisher Scientific, Inc.) The thermocycling conditions were as follows: $95^{\circ} \mathrm{C}$ for $3 \mathrm{~min}$; $95^{\circ} \mathrm{C}$ for $15 \mathrm{sec} ; 60^{\circ} \mathrm{C}$ for $15 \mathrm{sec} ; 72^{\circ} \mathrm{C}$ for $1 \mathrm{~min}(35$ cycles); and $72^{\circ} \mathrm{C}$ for $10 \mathrm{~min}$. The relative expression of genes was determined using the $2^{-\Delta \Delta \mathrm{cq}}$ method (19) with normalization to $36 \mathrm{~B} 4$ expression. The primer sequences were as follows: LH receptor (LHR) forward, 5'-AATGAGTCCATCACG CTGAAAC-3' and reverse, 5'-CCTGCAATTTGGTGG AAGAGA-3'; FSHR forward, 5'-CCTTGCTCCTGGTCT CCTTG-3' and reverse, 5'-CTCGGTCACCTTGCTATC TTG-3'; aromatase forward, 5'-ATGTTCTTGGAAATG CTGAACCC-3' and reverse, 5'-AGGACCTGGTATTGA AGACGAG-3'; peroxisome proliferator-activated receptor $\gamma$ (PPAR $\gamma$ ) forward, 5'-TTTTCCGAAGAACCATCCGATT-3' and reverse, 5'-ATGGCATTGTGAGACATCCCC-3'; and 34B4 forward, 5'-AAGCGCGTCCTGGCATTGTCT-3' and reverse, 5'-CCGCAGGGGCAGCAGTGGT-3'.

Isolation of granule cells. As described previously (20), ovaries were isolated from mice and the large and transparent follicles residing within were punctured to liberate granule cells into the medium, Dulbecco's modified Eagle's medium (DMEM)/nutrient mixture F-12 (F12; Gibco; Thermo Fisher Scientific, Inc.). Granule cells were mixed and collected via centrifugation at $100 \mathrm{x} \mathrm{g}$ at room temperature for 2 min. Cells were then suspended in DMEM/F12 complete medium containing $10 \%$ fetal bovine serum (Gibco; Thermo Fisher Scientific, Inc.) and seeded into 12-well plates at $3 \times 10^{5}$ cells/well for $48 \mathrm{~h}$. Medium was changed every $24 \mathrm{~h}$ to discard non-adherent granulosa cells and oocytes. Cells were further cultured at $37^{\circ} \mathrm{C}$ for $24 \mathrm{~h}$ for further experimentation.

Western blotting. Granule cells were homogenized in ice-cold lysis buffer [50 mM Tris- $\mathrm{HCl}(\mathrm{pH} 7.5), 150 \mathrm{mM} \mathrm{NaCl}, 2 \mathrm{mM}$ EDTA, $1 \%$ Nonidet P-40, $10 \mathrm{mM} \mathrm{Na}_{4} \mathrm{P}_{2} \mathrm{O}_{7} \cdot 10 \mathrm{H}_{2} \mathrm{O}$, and protein inhibitors including $100 \mathrm{mM} \mathrm{NaF}, 1 \mathrm{mM} \mathrm{Na} \mathrm{VO}_{4}$, $1 \mathrm{mM}$ phenylmethylsulfonyl fluoride, $10 \mathrm{mg} / \mathrm{ml}$ aprotinin, $10 \mathrm{mg} / \mathrm{ml}$ leupeptin; all components purchased from Aladdin Shanghai Biochemical Technology Co., Ltd., Shanghai, China) and boiled at $95^{\circ} \mathrm{C}$ for $5 \mathrm{~min}$ in $5 \mathrm{X}$ loading buffer 
A

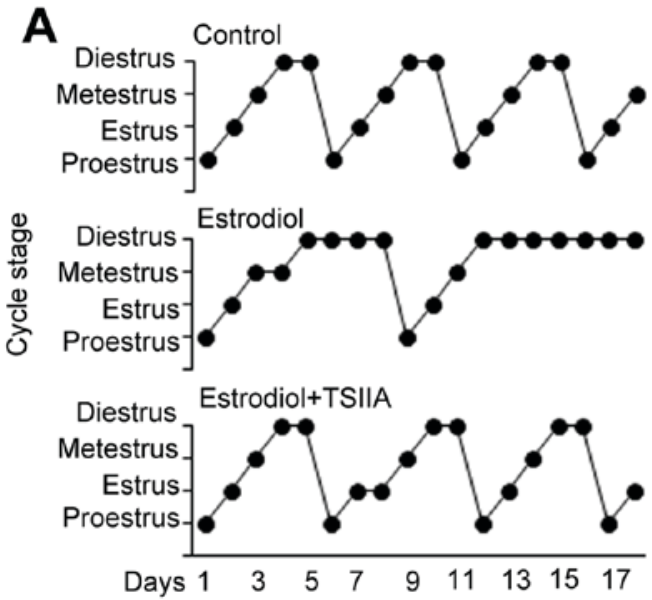

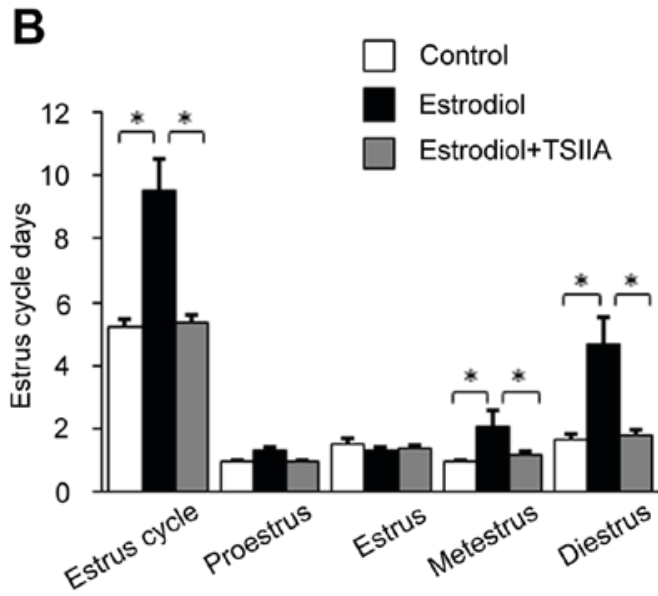

Figure 1. Effect of TSIIA on the disorder of the estrus cycle. (A) Estrus cycles of female mice were determined via toluidine blue staining of fluid obtained from vaginal lavage. Control $(n=8)$, Estradiol $(n=8)$, Estradiol + TSIIA $(n=8)$. (B) Estrus cycle lengths and stages, including proestrus, estrus, metestrus and diestrus were counted. The data were presented as the mean \pm standard error of the mean. ${ }^{*} \mathrm{P}<0.05$. TSIIA, Tanshinone IIA.

[250 mM Tris-HCl (pH 6.8), 10\% SDS, 0.5\% bromophenol blue, $50 \%$ glycerol, $5 \%$ mercaptoethanol; all components purchased from Aladdin Shanghai Biochemical Technology Co., Ltd.]. Protein concentration was assessed using a bicinchoninic acid protein assay kit (Pierce; Thermo Fisher Scientific, Inc.). Protein $(25 \mu \mathrm{g})$ was separated by $10 \%$ SDS-PAGE and transferred to polyvinylidene difluoride membranes. The membranes were incubated with rabbit anti-FSHR antibodies (1:1,000; cat. no. K002799P; Beijing Solarbio Science and Technology Co., Ltd., Beijing, China), anti-LHR antibodies (1:1,000; cat. no. sc-25828; Santa Cruz Biotechnology, Inc., Dallas, TX, USA), anti-aromatase antibodies (1:1,000; cat. no. PA1-21398; Invitrogen; Thermo Fisher Scientific, Inc.) and mouse anti- $\beta$-actin $(1: 1,000$; cat. no. CBL171-I; EMD Millipore, Billerica, MA, USA) at $4^{\circ} \mathrm{C}$ overnight. Membranes were then further incubated with horseradish peroxidase-conjugated goat anti-rabbit Immunoglobulin G (IgG; 1:5,000; cat. no. AP183P) or goat anti-mouse IgG (1:5,000; cat. no. AP192P; both EMD Millipore) for $2 \mathrm{~h}$ at room temperature. Membranes were visualized using the Tanon-5200 Chemiluminescence Imager (Tanon Science and Technology, Co., Ltd., Shanghai, China) with an ECL Western blotting substrate (EMD Millipore). ImageJ version 1.48 (National Institutes of Health, Bethesda, MA, USA) was used for the densitometry analysis of bands.

Cyclic adenosine monophosphate (cAMP) levels in granule cells. Following culture for $24 \mathrm{~h}$, non-adherent granule cells were removed and adherent cells were treated with FSH (100 IU/1; Ningbo Second Hormone Factory, Ningbo, China) for $10 \mathrm{~min}$ at $37^{\circ} \mathrm{C}$. Cells were then rinsed with cold PBS once and lysed using $0.1 \mathrm{M}$ hydrochloric acid for the protein concentration assay with total protein assay kit (cat. no. A045-4; Nanjing Jiancheng Bioengineering Institute, Nanjing, China). Whole cell lysate was centrifuged at $10,000 \mathrm{x}$ g at $4^{\circ} \mathrm{C}$ for $10 \mathrm{~min}$ and the supernatant was used for cAMP measurement using a cAMP ELISA kit (cat. no. 10R-2133; Fitzgerald Industries International, North Acton, MA, USA). Total cAMP amount was normalized to total protein amount in each sample to calculate cAMP level in cells.
Statistical analysis. Data were expressed as the mean \pm standard error of the mean. Data between groups were analyzed using one-way analysis of variance followed by Dunn's multiple comparisons test. $\mathrm{P}<0.05$ was considered to indicate a statistically significant result.

\section{Results}

TSIIA attenuates estrous cycle disorder in mice with PCOS. Normal control mice exhibited a regular estrous cycle of 5 days on average (Fig. 1A). The entire estrous cycle comprises four phases, which includes proestrus, estrus, metestrus and diestrus. Neonatal estradiol-treatment increased metestrus and diestrus compared with control mice, which contributed to the prolongation of the estrous cycle to $\sim 9$ days (Fig. 1A and B). TSIIA treatment significantly reduced entire estrous cycle, metestrus and diestrus and led to a slight increase in the estrous period (Fig. 1A and B).

Effects of TSIIA on ovarian morphology in mice with PCOS. Body weight and ovary weight in each group were assayed following sacrifice. The results revealed that neonatal estradiol treatment increased body and ovarian weight in mice (Fig. 2A and B). The increase in ovarian weight was more pronounced than that of body weight, and the ratio of ovary to body weight also significantly increased following estradiol treatment (Fig. 2C). TSIIA treatment reversed the estradiol-induced increase in body weight, ovary weight and the ratio of ovary to body weight (Fig. 2A-C).

Pathological changes in murine ovaries were assessed via $H \& E$ staining. In congruence with a previous study (14), multiple enlarged follicles were observed in the ovaries of estradiol-treated mice in the present study (Fig. 2D), which mimicked PCOS. Neonatal estradiol-treatment reduced the number of large antral follicles, preovulatory follicles and corpus luteum, but increased the number of atretic cyst-like follicles (Fig. 2D), which were also similar to changes typically observed in PCOS (6). TSIIA treatment reversed the observed pathological change in the number of follicles (Fig. 2D). 

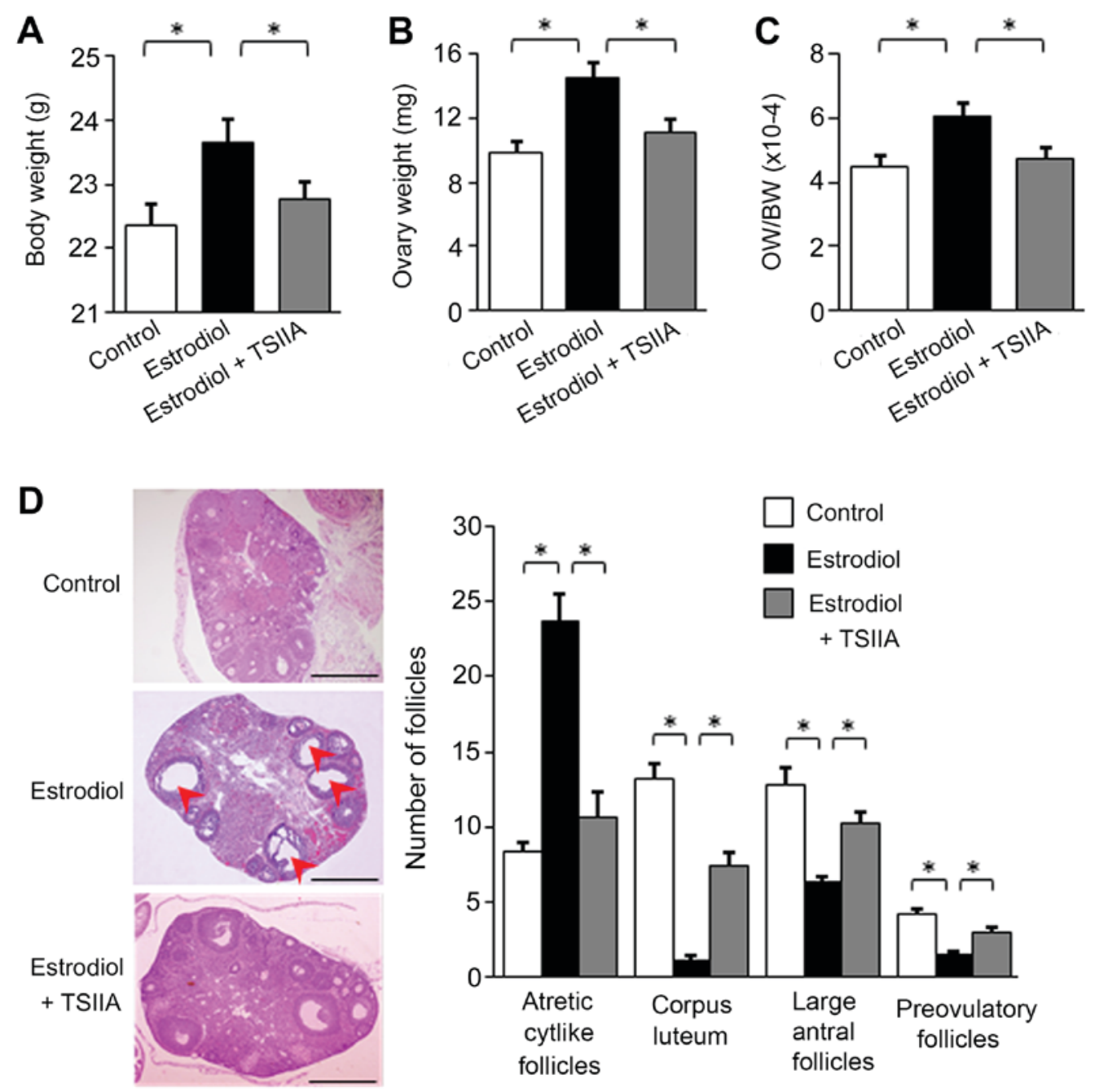

Figure 2. Effect of TSIIA on pathological changes induced by estradiol treatment. (A) BW and (B) OW in each group were assayed in mice following sacrifice at 12-weeks of age. Control $(n=8)$, Estradiol $(n=8)$, Estradiol + TSIIA $(n=8)$. (C) OW/BW ratio. (D) Ovarian tissue was stained with hematoxylin and eosin. The number of atretic cyst-like follicles (red arrows), corpus luteums, large antral follicles and preovulatory follicles were counted. Scale bar, $500 \mu \mathrm{m}$. Data are expressed as the mean \pm standard error of the mean. ${ }^{*} \mathrm{P}<0.05$. TSIIA, Tanshinone IIA; OW, ovarian weight, BW, body weight.

TSIIA regulates serum hormone levels in mice with PCOS. Ovarian structure and function is primarily regulated by serum hormones (6). Thus, levels of FSH, LH, estradiol, T and P in murine serum were assayed. As presented in Fig. 3A-C, serum levels of LH,FSH and the ratio of LH to FSH were not significantly affected by neonatal estradiol-treatment nor by TSIIA. However, serum estradiol was reduced and $\mathrm{T}$ levels were elevated following neonatal estradiol treatment, which were each reversed when further treated with TSIIA (Fig. 3D and E). Serum P levels were also reduced following neonatal estradiol-treatment, but TSIIA demonstrated no significant effect.

Effect of TSIIA on LHR, FSHR, aromatase and PPAR $\gamma$ expression in the ovaries of mice with PCOS. Neonatal mice treated with estradiol exhibited significantly lower mRNA levels of aromatase, the key enzyme for the transferal of $\mathrm{T}$ to estradiol in ovaries (21). However, mRNA levels of ovarian aromatase recovered following TSIIA treatment. FSHR, LHR and PPAR $\gamma$ function to regulate the expression of aromatase in the ovary (22-24); however, only FSHR exhibited significantly lower mRNA levels following neonatal estradiol treatment, which was recovered by TSIIA (Fig. 4A).
In ovarian granule cells (Fig. 4B-D) of neonatal estradiol-treatment mice, western blot analysis revealed lower FSHR and aromatase protein levels, which were improved following TSIIA treatment (Fig. 4B).

The FSH-induced cAMP levels in granule cells isolated from estradiol-treated mice were significantly lower than that from control mice, whereas TSIIA treatment elevated the granular cAMP level in estradiol-treated group similar to control group (Fig. 4C).

The results also demonstrated that granular FSHR and aromatase mRNA levels in estradiol treated group were significantly lower than in the control group, but were reversed following TSIIA treatment (Fig. 4D).

\section{Discussion}

The present study revealed the therapeutic effect of TSIIA, a component isolated from Salvia miltiorrhiza, on murine PCOS induced by the neonatal treatment of estradiol.

In a previous study (25), PCOS was successfully replicated in mice via neonatal treatment with estradiol. This was determined as mice exhibited a prolonged diestrus phase, 
A
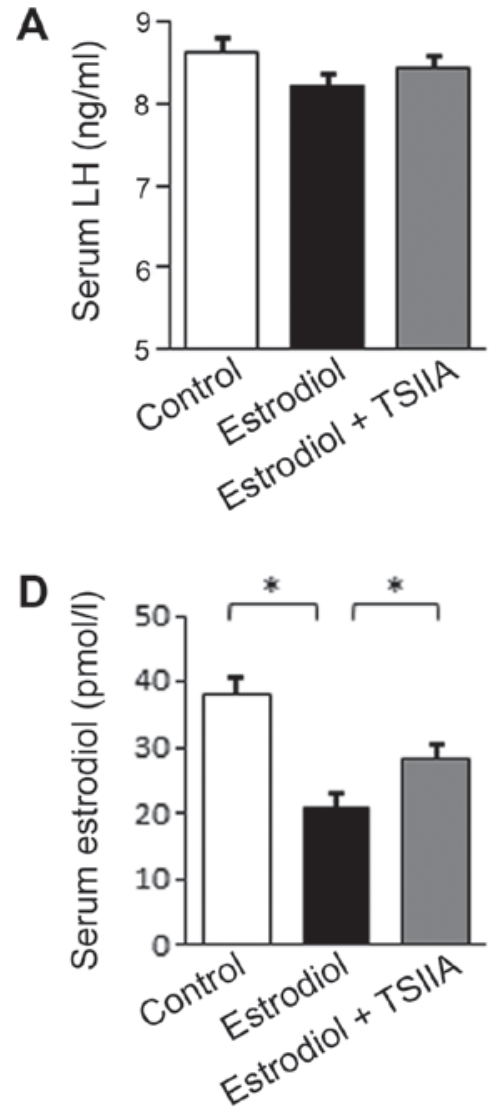

B

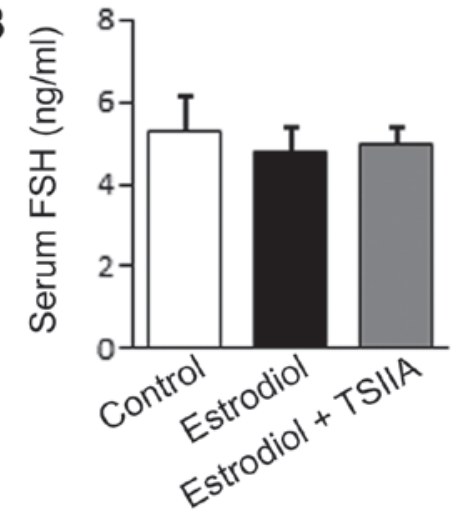

E

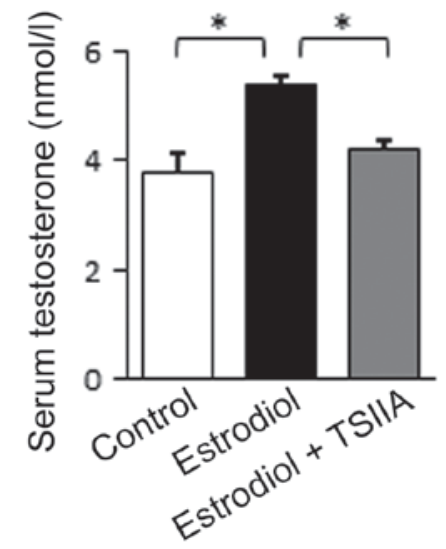

C


Figure 3. Effect of TSIIA on serum hormones levels. Serum levels of (A) LH, (B) FSH, (C) the LH/FSH ratio, (D) estradiol, (E) testosterone and (F) progesterone were determined following modeling by estradiol and subsequent 4-week TSIIA treatment. Control ( $\mathrm{n}=8$ ), Estradiol $(\mathrm{n}=8)$, Estradiol + TSIIA ( $\mathrm{n}=8)$. Data are expressed as the mean \pm standard error of the mean. ${ }^{*} \mathrm{P}<0.05$. TSIIA, Tanshinone IIA; LH, luteinizing hormone; FSH, follicle stimulating hormone.

enlarged ovaries, a thickened theca layer, a decreased number of corpus luteums and preovulatory follicles, an increased number of atretic follicles and multiple cysts in ovaries, which are characteristics similar to those in women with PCOS (19). In the current study, estrus cycles were returned to normal status following TSIIA treatment and this also reversed the pathological change of murine ovaries with estradiol-induced PCOS.

The alteration of granulosa cell hormonal equilibrium is a cause of disturbed follicular development and maturation that results in the premature arrest of follicular growth in PCOS $(26,27)$. Estrogen suppresses granulosa and luteal cell apoptosis $(28,29)$ and triggers the release of FSH and $\mathrm{LH}$, promoting follicle maturation and ovulation (30). An excess of intraovarian androgen leads to follicular atresia (28), as it activates primordial follicles via the phosphatidylinositol-3-kinase/protein kinase B/Forkhead box O3a pathway and inhibits growth differentiation factor 9 expression in oocytes, resulting in the arrest of preantral follicle development (31).

In the present study, neonatal treatment of estradiol decreased serum estradiol but increased serum $\mathrm{T}$ levels, which may lead to pathological and functional changes in the ovary. Thus, the therapeutic effect of TSIIA observed in the current study may be the result of disordered estradiol and T, as other hormones affecting follicle maturation, including LH, FSH and $\mathrm{P}$, were either not changed or not reversed following TSIIA therapy.
The hormonal balance between estrogen and androgen is primarily sustained by the normal function of granulosa cells, which contain a series of enzymes converting $\mathrm{T}$ to estradiol. Disorder in the activity or expression of aromatase, the key enzyme catalyzing the conversion of $\mathrm{T}$ to estradiol, induces excessive androgen accumulation in serum (32). Thus, aromatase mRNA levels of the ovaries and granule cells were determined in the current study. It was demonstrated that they were reduced by neonatal estradiol-treatment, but ameliorated following TSIIA therapy. This implies that TSIIA targets the expression of aromatase to revise the T/estradiol balance in mice.

The transcription of aromatase is regulated by the LH, FSH and PPAR $\gamma$ pathways (22-24). LH and FSH mediate the downor upregulation of aromatase via LHR and FSHR, respectively. Since the serum levels of LH and FSH were not affected by neonatal estradiol treatment or TSIIA in the current study, the expression of LHR and FSHR should predict the transcription of aromatase. FSHR mRNA levels were reduced by neonatal estradiol treatment, which was then ameliorated by TSIIA. However, neither LHR nor PPAR $\gamma$ expression was affected. The results indicate that the downregulation of FSHR induced PCOS in the present murine model and that TSIIA exerted its therapeutic effect by targeting FSHR expression. Furthermore, the present study revealed that estradiol treatment reduced FSHR and aromatase protein levels, thus inhibiting FSH-induced cAMP elevation in granule cells isolated from mice. TSIIA treatment also ameliorated FSHR expression and 


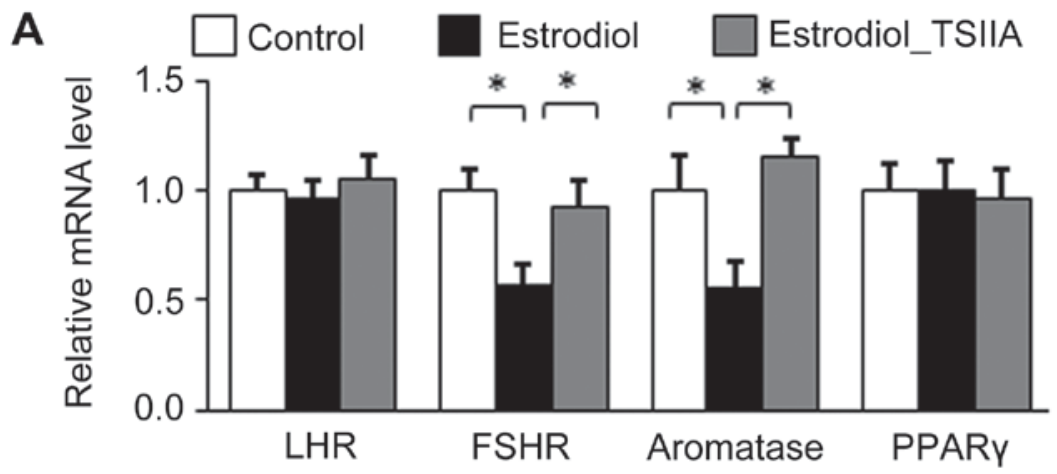

B
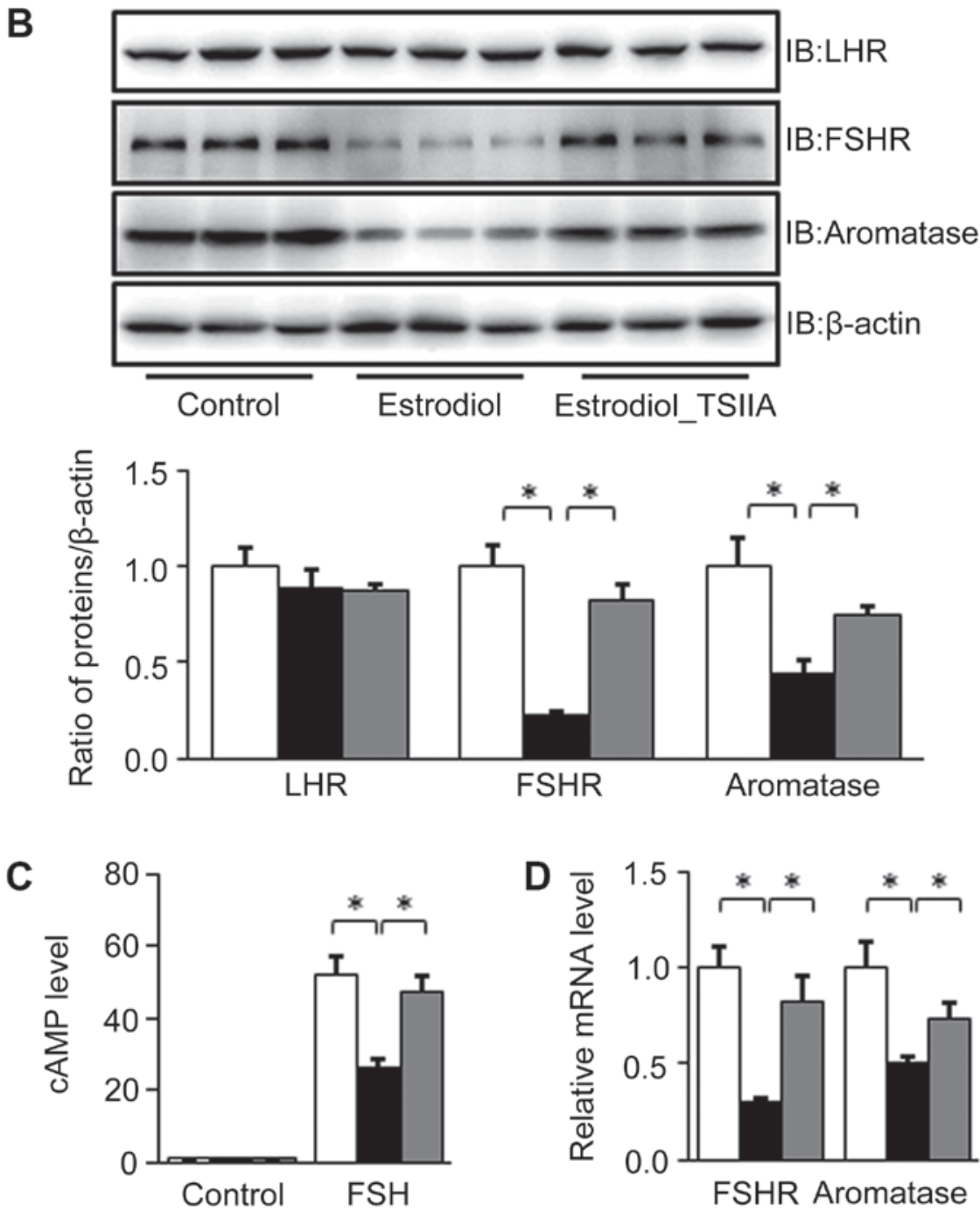

Figure 4. Effect of TSIIA on the mRNA expression of genes associated with ovarian hormone levels. (A) mRNA levels of LHR, FSHR, aromatase and PPAR $\gamma$ in ovaries ( $\mathrm{n}=8$ for each group). Granule cells were isolated from Estradiol, Estradiol+TSIIA and control mice. (B) FSHR, LHR and aromatase protein levels in granule cells. (C) cAMP levels in granule cells treated with/without FSH. (D) mRNA levels of FSHR and aromatase in granule cells. Data are expressed as the mean \pm standard error of the mean. "P<0.05. TSIIA, Tanshinone IIA; LHR, luteinizing hormone receptor; FSHR, follicle stimulating hormone receptor; PPAR $\gamma$, peroxisome proliferator-activated receptor $\gamma$; cAMP, cyclic adenosine monophosphate.

an FSH induced cAMP increase. As a result, TSIIA primarily affected the ovarian expression of FSHR, which improved ovarian function.

In summary, the present study revealed that PCOS induced by neonatal exposure to estradiol in mice is attenuated by TSIIA treatment. The therapeutic effect of TSIIA is likely due to the recovery of FSHR expression in the ovary, thus leading to elevated serum levels of estradiol and $\mathrm{T}$, an improvement in the structure of ovaries and the attenuation of the disordered estrous cycle. The current study revealed a novel monomer (TSIIA), which may be utilized to further the application of natural drugs in the treatment of PCOS to reduce the side effects of hormone drugs. However, the present study was limited by the lack of a positive control group and intensive studies in granule cells. 
Further study is therefore required to assess the mechanism of how TSIIA upregulates FSHR in granule cells.

\section{Acknowledgements}

The authors would like to thank Professor Liang Sheng from Nanjing Medical University (Nanjing, China) for assisting with the experimental design and modifications to the manuscript.

\section{Funding}

The present study was supported by the Natural Science Foundation of China (grant nos. 81473713 and 81774354), Jiangsu Provincial Hospital of TCM (grant no. Y18070) and Leading talents of Chinese medicine in Jiangsu province (grant no. SLJ0202).

\section{Availability of data and materials}

All data and materials supported the results of the present study are available in the published article.

\section{Authors' contributions}

HFZ was responsible for the experimental design and drafting the manuscript. JJ, QYH, WWX, WJZ, BL, JL and WW performed the experiments and data analysis. HFZ reviewed the manuscript. All authors gave final approval of the version to be published.

\section{Ethics approval and consent to participate}

Animal care and experimental procedures were approved by and performed in accordance with the guidelines of the Animal Care and Ethics Committee of Affiliated Hospital of Nanjing University of Chinese Medicine.

\section{Patient consent for publication}

Not applicable.

\section{Competing interests}

The authors declare that they have no competing interests.

\section{References}

1. Fauser BC, Tarlatzis BC, Rebar RW, Legro RS, Balen AH, Lobo R, Carmina E, Chang J, Yildiz BO, Laven JS, et al: Consensus on women's health aspects of polycystic ovary syndrome (PCOS): The Amsterdam ESHRE/ASRM-Sponsored 3rd PCOS Consensus Workshop Group. Fertil Steril 97: 28-38. e25, 2012.

2. Abbott DH, Tarantal AF and Dumesic DA: Fetal, infant, adolescent and adult phenotypes of polycystic ovary syndrome in prenatally androgenized female rhesus monkeys. Am J Primatol 71: 776-784, 2009.

3. Jones GL, Hall JM, Balen AH and Ledger WL: Health-related quality of life measurement in women with polycystic ovary syndrome: A systematic review. Human Reprod Update 14: 15-25, 2008.

4. Jeanes YM and Reeves S: Metabolic consequences of obesity and insulin resistance in polycystic ovary syndrome: Diagnostic and methodological challenges. Nutr Res Rev 30: 97-105, 2017.
5. Lauretta R, Lanzolla G, Vici P, Mariani L, Moretti C and Appetecchia M: Insulin-sensitizers, polycystic ovary syndrome and gynaecological cancer risk. Int J Endocrinol 2016: 8671762, 2016.

6. De Leo V, Musacchio MC, Cappelli V, Massaro MG, Morgante G and Petraglia F: Genetic, hormonal and metabolic aspects of PCOS: An update. Reprod Biol Endocrinol 14: 38, 2016.

7. Rocca ML, Venturella R, Mocciaro R, Di Cello A, Sacchinelli A, Russo V, Trapasso S, Zullo F and Morelli M: Polycystic ovary syndrome: Chemical pharmacotherapy. Expert Opin Pharmacother 16: 1369-1393, 2015.

8. Saeed R, Akram J, Changezi HU and Saeed M: Treatment of hirsutism in polycystic ovarian syndrome with Diane, $50 \mathrm{mcg}$ ethinyl estradiol and $2 \mathrm{mg}$ cyproterone acetate. Specialist 9: 109-112, 1993.

9. Jing Z, Liang-Zhi X, Tai-Xiang W, Ying T and Yu-Jian J: The effects of Diane-35 and metformin in treatment of polycystic ovary syndrome: An updated systematic review. Gynecol Endocrinol 24: 590-600, 2008.

10. Huang Y, Li W, Wang CC, Wu X and Zheng J: Cryptotanshinone reverses ovarian insulin resistance in mice through activation of insulin signaling and the regulation of glucose transporters and hormone synthesizing enzymes. Fertil Steril 102: 589-596.e4, 2014.

11. Li P, Wang GJ, Li J, Hao HP and Zheng CN: Identification of tanshinone IIA metabolites in rat liver microsomes by liquid chromatography-tandem mass spectrometry. J Chromatogr A 1104: 366-369, 2006.

12. Chen $\mathrm{Z}$ and $\mathrm{Xu} \mathrm{H}$ : Anti-inflammatory and immunomodulatory mechanism of tanshinone IIA for atherosclerosis. Evid Based Complement Alternat Med 2014: 267976, 2014.

13. Wang ZY, Liu JG, Li H and Yang HM: Pharmacological effects of active components of chinese herbal medicine in the treatment of Alzheimer's disease: A review. Am J Chin Med 44: 1525-1541, 2016.

14. Chapman JC, Min SH, Freeh SM and Michael SD: The estrogen-injected female mouse: New insight into the etiology of PCOS. Reprod Biol Endocrinol 7: 47, 2009.

15. Goldman JM, Murr AS and Cooper RL: The rodent estrous cycle: Characterization of vaginal cytology and its utility in toxicological studies. Birth Defects Res B Dev Reprod Toxicol 80: 84-97, 2007.

16. Caldwell AS, Middleton LJ, Jimenez M, Desai R, McMahon AC, Allan CM, Handelsman DJ and Walters K: Characterization of reproductive, metabolic, and endocrine features of polycystic ovary syndrome in female hyperandrogenic mouse models. Endocrinology 155: 3146-3159, 2014.

17. Myers M, Britt KL, Wreford NG, Ebling FJ and Kerr JB: Methods for quantifying follicular numbers within the mouse ovary. Reproduction 127: 569-580, 2004.

18. Glidewell-Kenney C, Hurley LA, Pfaff L, Weiss J, Levine JE and Jameson JL: Nonclassical estrogen receptor alpha signaling mediates negative feedback in the female mouse reproductive axis. Proc Natl Acad Sci USA 104: 8173-8177, 2007.

19. Livak KJ and Schmittgen TD: Analysis of relative gene expression data using real-time quantitative PCR and the 2(-Delta Delta C(T)) method. Methods 25: 402-408, 2001.

20. Liang N, Xu Y, Yin Y, Yao G, Tian H, Wang G, Lian J, Wang Y and Sun F: Steroidogenic Factor-1 is required for TGF- $\beta 3$-mediated $17 \beta$-estradiol synthesis in mouse ovarian granulosa cells. Endocrinology 152: 3213-3225, 2011.

21. Simpson ER and Davis SR: Minireview: Aromatase and the regulation of estrogen biosynthesis-some new perspectives. Endocrinology 142: 4589-4594, 2001.

22. Fan W, Yanase T, Morinaga H, Mu YM, Nomura M, Okabe T, Goto $\mathrm{K}$, Harada $\mathrm{N}$ and Nawata $\mathrm{H}$ : Activation of peroxisome proliferator-activated receptor-gamma and retinoid X receptor inhibits aromatase transcription via nuclear factor-kappaB. Endocrinology 146: 85-92, 2005.

23. Komar CM: Peroxisome proliferator-activated receptors (PPARs) and ovarian function-implications for regulating steroidogenesis, differentiation, and tissue remodeling. Reprod Biol Endocrinol 3: 41, 2005.

24. Komar CM, Braissant O, Wahli W and Curry TE Jr: Expression and localization of PPARs in the rat ovary during follicular development and the periovulatory period. Endocrinology 142: 4831, 2001.

25. van Houten EL and Visser JA: Mouse models to study polycystic ovary syndrome: A possible link between metabolism and ovarian function. Reprod Biol 14: 32-43, 2014. 
26. Lombardi LA, Simãues RS, Maganhin CC, Baracat MC, Silva-Sasso GR, Florencio-Silva R, Soares JM Jr and Baracat EC: Immunohistochemical evaluation of proliferation, apoptosis and steroidogenic enzymes in the ovary of rats with polycystic ovary. Rev Assoc Med Bras (1992) 60: 349-356, 2014.

27. Salilew-Wondim D, Wang Q, Tesfaye D, Schellander K, Hoelker M, Hossain MM and Tsang BK: Polycystic ovarian syndrome is accompanied by repression of gene signatures associated with biosynthesis and metabolism of steroids, cholesterol and lipids. J Ovarian Res 8: 24, 2015.

28. Billig H, Furuta I and Hsueh AJ: Estrogens inhibit and androgens enhance ovarian granulosa cell apoptosis. Endocrinology 133: 2204-2212, 1993.

29. Goodman SB, Kugu K, Chen SH, Preutthipan S, Tilly KI, Tilly JL and Dharmarajan AM: Estradiol-mediated suppression of apoptosis in the rabbit corpus luteum is associated with a shift in expression of bcl-2 family members favoring cellular survival. Biol Reprod 59: 820-827, 1998.
30. Caligioni CS: Assessing reproductive status/stages in mice. Curr Protoc Neurosci 4: Appendix 4I, 2009.

31. Yang JL, Zhang CP, Li L, Huang L, Ji SY, Lu CL, Fan CH, Cai H, Ren Y, Hu ZY, et al: Testosterone induces redistribution of forkhead box-3a and down-regulation of growth and differentiation factor 9 messenger ribonucleic acid expression at early stage of mouse folliculogenesis. Endocrinology 151: 774-782, 2010.

32. Capellino S, Straub RH and Cutolo M: Aromatase and regulation of the estrogen-to-androgen ratio in synovial tissue inflammation: Common pathway in both sexes. Ann N Y Acad Sci 1317: 24-31, 2014.

This work is licensed under a Creative Commons Attribution-NonCommercial-NoDerivatives 4.0 International (CC BY-NC-ND 4.0) License. 